\title{
Optical Fibre Nanowires and Related Structures
}

\author{
Gilberto Brambilla, Fei Xu \\ Optoelectronics Research Centre, University of Southampton, Southampton, SO17 1BJ, United Kingdom \\ Tel: (44)238059 7673, Fax: (44)23 8059 3149, e-mail:gb2@orc.soton.ac.uk
}

\begin{abstract}
The fabrication of optical fibre nanowires by flame-brushing represents a top-down method to reliably manufacture the longest, most robust nanowires from silica and compound glasses. Small surface roughness and long-length diameter homogeneity provide the low-level of loss needed for optical applications. A considerable fraction of the transmitted power propagates in the evanescent field outside of a nanowire allowing the prompt fabrication of high-Q resonators and several sensors.
\end{abstract}

Keywords: Optical nanowires, optical fibres, fabrication, sensor.

\section{INTRODUCTION}

Nanowires have been manufactured from a variety of materials [1] using several different methods, such as electron beam lithography [2], bottom-up techniques like chemical/physical vapor deposition [3] and top-down processes like fibre drawing [4,5]. The use of top-down techniques for the manufacture of nanowires from optical fibres provides the longest, most uniform and robust nanowires [5]. Moreover, the low optical loss associated to the small surface roughness allows the use of nanowires for transmitting light [4-6].

Optical fibre nanowires and sub-wavelength wires (OFNSW) are attractive for a variety of promising fibre optic applications because of their enabling optical and mechanical properties. In particular they allow for: (a) large evanescent fields outside their physical boundary, (b) high nonlinearity, (c) strong confinement, (d) lowloss interconnection to fiberized components. OFNSW are fabricated by adiabatically stretching optical fibres and thus preserve the original optical fibre dimensions at their input and output allowing ready splicing to standard fibres. Because of the large evanescent field, when nanowires are coiled the mode propagating in the nanowire is interfering with itself to give a resonator.

In the next sections the latest results on the manufacture of OFNSW from silica and compound glasses are presented, with a particular stress to their optical and mechanical characterization. High-Q factor resonators and their application for biosensors are proposed.

\section{FABRICATION}

Three methods have recently emerged for the top-down fabrication of OFNSW from optical fibres:

a) A sapphire rod heated by a flame [4].

b) A microfurnace made by a hollow sapphire cylinder heated by a $\mathrm{CO}_{2}$ laser [7].

c) The flame-brushing technique previously used to manufacture tapers/couplers from optical fibres [5,8-10].

A summary of the properties of these methodologies is reported in table 1 .

Table 1: Specifications of top-down fabrication techniques.

\begin{tabular}{|l|c|c|c|c|}
\hline & & $\begin{array}{c}\text { (a): sapphire } \\
\text { rod + flame }\end{array}$ & $\begin{array}{c}\text { (b): sapphire cylinder } \\
+\mathrm{CO}_{2} \text { laser }\end{array}$ & $\begin{array}{c}\text { (c): flame } \\
\text { brushing }\end{array}$ \\
\hline Minimum loss at $\mathrm{r} \sim 430 \mathrm{~nm}, \lambda \sim 1.55 \mu \mathrm{m}$ & {$[\mathrm{dB} / \mathrm{mm}]$} & $\sim 10^{-1}[4]$ & $\sim 10^{-1}[7]$ & $\sim 10^{-3}[10]$ \\
\hline Minimum radius nanowires & {$[\mathrm{nm}]$} & $10[12]$ & $\sim 50[7]$ & $\sim 30[10]$ \\
\hline Maximum length & {$[\mathrm{mm}]$} & tens $[4]$ & few $[7]$ & $\sim 110[5]$ \\
\hline Uniformity & $10^{-6}$ & $2[4]$ & - & $0.5[5]$ \\
\hline Pigtails & & 1 & 2 & 2 \\
\hline
\end{tabular}

In (a) a pre-tapered fibre is wound on a hot sapphire tip, and drawn further to submicron diameters. Although this methodology has been used to manufacture extremely small-diameter wires, it presents several drawbacks: complex fabrication procedure, relatively high loss (at least ten times more than technique c) and single-sided access to the nanowires.

The process (b) involves inserting an optical fibre into a sapphire capillary tube, which is heated by a $\mathrm{CO}_{2}$ laser beam. This method is very fast, provides nanowires with very short tapers and radii as small as 50nm. Drawbacks of this process include the high loss and the short length of drawn nanowires.

The authors gratefully acknowledge the financial support by the EPSRC, under the standard research grant EP/C00504X/1. 
(c) is based on a small flame moving under an optical fibre which is stretched (for this reason it is also called flame-brushing). The control of the flame movement and the fibre stretch can define the taper shape to an extremely high degree of accuracy. Nanowires with radii as small as 30nm have been demonstrated [10]. This methodology provides the longest and most uniform nanowires [5] with the lowest measured losses [8-10]. Moreover, it provides access to the nanowires with two pigtails.

Nanowires longer than 110mm have been manufactured using the flame-brushing technique [5]. Figure 1 shows pictures of OFNSW fabricated by this technique. OFNSW size and uniformity have been evaluated using a scanning electron microscope (SEM): size homogeneity has often been within the instrument resolution, while uniformity (defined as the ratio between diameter fluctuations and nanowire length) was better than $10^{-7}$.

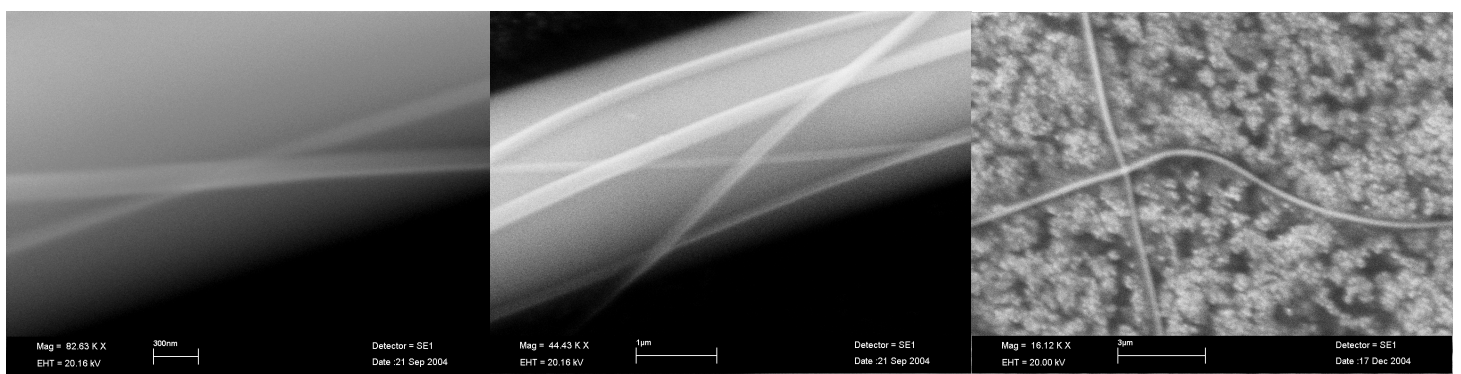

Figure 1. SEM pictures of silica and compound glass OFNSWs. The smallest radius is r 30nm.

By using the flame-brushing technique with a microheater as a heat source, OFNSW have been manufactured from a variety of compound-glasses [11], including telluride, bismuth-silicate, lead-silicate and chalcogenide fibres.

\section{OPTICAL AND MECHANICAL CHARACTERIZATION}

In order to measure the total optical loss $\alpha$ of the OFNSW, light at wavelength $\lambda=1550 \mathrm{~nm}$ was launched into the OFNSW pigtail and the transmitted signal was measured by an InGaAs detector. Figure 2 summarizes recorded losses in OFNSW made from telecom optical fibres versus OFNSW radius for $\lambda=1550 \mathrm{~nm}$. Losses achieved with other fabrication methods reported in the literature are also shown for comparison. It is clear that flame brushing provides lower losses compared to other fabrication techniques.

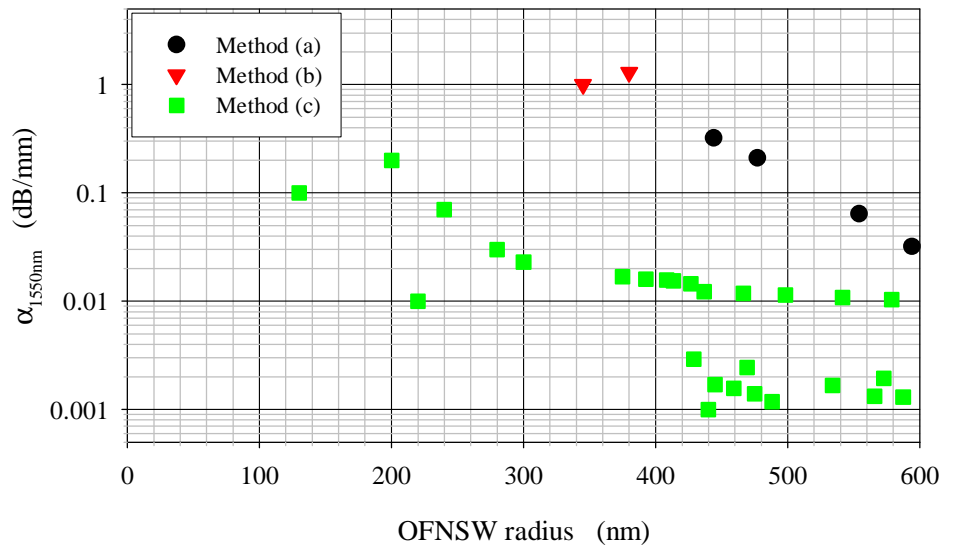

Figure 2. Comparison of optical loss at $\lambda=1550 \mathrm{~nm}\left(\alpha_{1550 \mathrm{~nm}}\right)$ for OFNSWs manufactured by different methods (see table 1); data for $(a)$ and $(b)$ were extracted from references 4 and 7 , respectively.

The mechanical strength was measured by adding weights at the bottom extremity of an OFNSW held vertically until fracture occurs. Measurements have been performed on OFNSW with $\mathrm{r}=375 \mathrm{~nm}$. The typical stress $\sigma_{\text {fract }}$ (defined as the ratio between weight at fracture and OFNSW section) was in the range of $\sigma_{\text {fract }} \sim 10-11 \mathrm{GPa}$. As before, it is interesting to note that this value seems considerably higher than that achieved for samples manufactured by method (a), for which the reported tensile strength was $2.5-5 \mathrm{GPa}$ [4]. It is well known that tensile strength in optical fibres is strongly dependent on surface quality; the better mechanical performance of the flame brushing technique could then be explained by the better surface quality (as also shown in table 1). 


\section{OPTICAL PROPAGATION, CONFINEMENT AND RESONATORS}

In OFNSW the radius $r$ can be considerably smaller than the wavelength $\lambda$ of the propagating beam, therefore the description of light propagation requires an exact solution of Maxwell's equations. Figure 3a shown the fullwidth-at-half-maximum of the amplitude of the propagating beam as calculated in OFNSW manufactured from silica (refractive index at $\lambda=1550 \mathrm{~nm} n \sim 1.44)$, lead-silicate $(\mathrm{n} \sim 1.76)$ and bismuth silicate $(\mathrm{n} \sim 2.01)$. It is interesting to note that after reaching a minimum, the beam expand considerably and in this regime much of the optical power can propagate outside the nanowire physical boundary: for a silica OFNSW with $\mathrm{r} \sim 130 \mathrm{~nm}$, at $\lambda \sim 1550 \mathrm{~nm}$ more than $99.99 \%$ of the modal power propagates outside of the wire and the intensity decreases to $10 \%$ of its maximum value at a distance of $\sim 70 \mu \mathrm{m}$ from the surface.

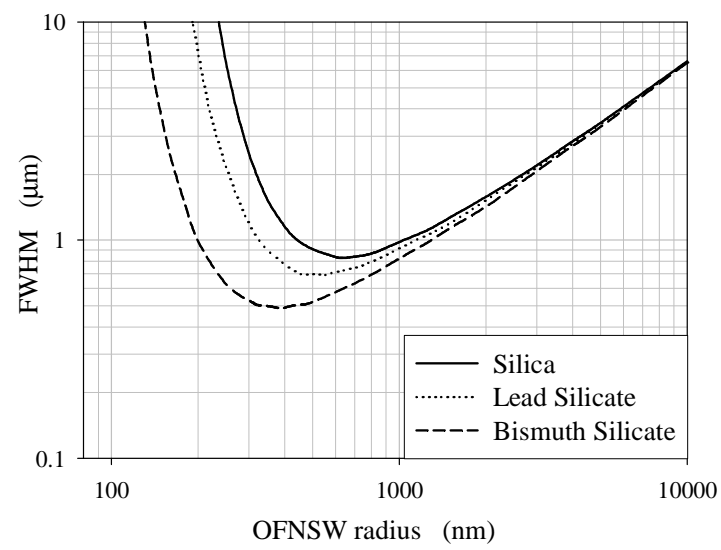

Figure $3 a$. Relation between the beam full width at half maximum at $\lambda=1550 \mathrm{~nm}$ ( FWHM) of fundamental mode $\left(H E_{11}\right)$ and the OFNSW radius for three selected materials.

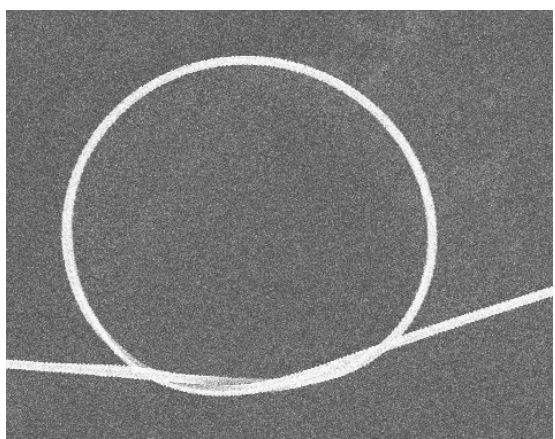

Figure 3b. Knot high-Q microcoil resonator manufactured from an OFNSW. The resonator radius is approximately $R \sim 45 \mu \mathrm{m}$.

Since the FWHM is larger than the OFNSW physical dimension, coiling allows the propagating mode to be coupled back into the OFNSW after a round trip. This generates a very compact resonator with extremely high$\mathrm{Q}$. As an example, figure $3 \mathrm{~b}$ shows a knot resonator with radius $\mathrm{R} \sim 45 \mu \mathrm{m}$. Coil resonators with Q-factors in excess of $10^{5}$ have been demonstrated.

\section{A SENSOR}

The high Q-factors achievable in OFNSW resonators guarantees large storage times for light within the resonator mode and hence long interaction times with any medium in contact with the mode propagating inside the coil. This makes the OFNSW resonator an ideal candidate for sensing applications. Figure 4a shows a possible design for the sensor: an OFNSW is embedded in Teflon and a channel is left in the device centre for the delivery of the chemical/analyte. The embedding process is aimed to increase the device robustness, stabilize its geometry and therefore its optical spectrum.

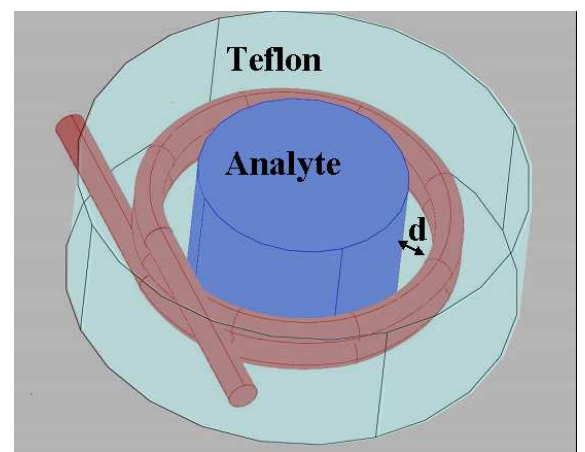

Figure 4a. Schematic of an embedded OFNSW microcoil resonator used as a biosensor.

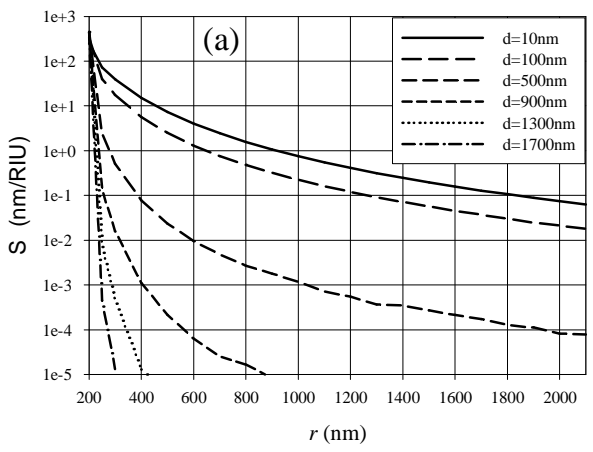

Figure 4b. Device sensitivity $S$ versus OFNSW radius $r$ as a function of the coating thickness $d$ 
If the OFNSW optical input is constant in time, changes in the analyte manifest first in a change of the effective index $n_{\text {eff }}$ of the propagating mode and then in the transmission spectrum. The incremental change of resonant wavelength per unit of refractive index change in the analyte is defined as homogeneous sensitivity $\mathrm{S}$ :

$$
S=\frac{\partial \lambda_{\text {res }}}{\partial n_{a}}=\frac{\lambda_{\text {res }}}{n_{\text {eff }}} \frac{\partial n_{\text {eff }}}{\partial n_{a}}
$$

Figure $4 \mathrm{~b}$ shows the expected device sensitivity at $\lambda=600 \mathrm{~nm}$ for several OFNSW dimensions as a function of the distance $\mathrm{d}$ between the OFNSW and the aqueous analyte. The refractive index of OFNSW, analyte and Teflon were assumed to be $n_{\text {OFNSW }}=1.451, n_{a}=1.332$ and $n_{t}=1.311$, respectively. It is interesting to note that the sensitivity is high when there is a big overlap between the mode propagating in the OFNSW and the analyte. This happens for a small $\mathrm{r}$ and/or a small $\mathrm{d}$. S as high as 700 can be achieved for small OFNSW radii. This value is comparable to the values reported in literature for other biosensors like the hollow-core antiresonant reflecting optical waveguide (ARROW) [13] or gratings [14]. However, the resonance bandwidth in OFNSW is orders of magnitude narrower than in gratings or ARROW devices, meaning that the minimum detectable quantity of analyte is orders of magnitude smaller for the OFNSW.

\section{CONCLUSIONS}

OFNSW fabricated by the flame brushing technique have been shown to exhibit extremely low optical loss and good mechanical properties. A considerable fraction of the bounded mode propagates outside the physical boundary for small radii. This can be exploited to make microcoil resonators with compact design and high Qfactors. A biosensor designed embedding an OFNSW resonator has been proposed: high sensitivity and extremely low minimum-detectable-limit have been predicted for small OFNSW radii.

\section{ACKNOWLEDGEMENTS}

The authors gratefully acknowledge the help of Dr. N. Broderick with the software package COMSOL, initiating discussions with Prof. D. J. Richardson and useful interactions with Dr. P. Horak

\section{REFERENCES}

[1] Z.L. Wang: Nanowires and Nanobelts: Materials, Properties and Devices (2 volumes), Ed., New York: Springer, 2006.

[2] J. Chen, M.A. Reed, A.M. Rawlett, J.M. Tour: Large On-Off Ratios and Negative Differential Resistance in a Molecular Electronic Device, Science, vol. 286, pp. 1550-1552. 1999.

[3] M. Yazawa, M. Koguchi, A. Muto, M. Ozawa, K. Iruma: Effect of one monolayer of surface gold atoms on the epitaxial growth of InAs nanowhiskers, Appl. Phys. Lett., vol. 61, pp. 2051-2054, 1992.

[4] L.M. Tong, et al.: Subwavelength-diameter silica wires for low-loss optical wave guiding, Nature, vol. 426, pp. 816-819, 2003.

[5] G. Brambilla, V. Finazzi, D.J. Richardson: Ultra-low-loss optical fiber nanotapers, Opt. Express, vol. 12, pp. 2258-2263, 2004.

[6] D. Marcuse, R.M. Derosier: Mode conversion caused by diameter changes of a round dielectric waveguide, Bell Syst. Tech. J., vol. 48, pp. 3217-3232, 1969.

[7] M. Sumetsky, Y. Dulashko, A. Hale: Fabrication and study of bent and coiled free silica nanowires: Selfcoupling microloop optical interferometer, Opt. Express, vol. 12, pp. 3521-3531, 2004.

[8] S.G. Leon-Saval, T.A. Birks, W.J. Wadsworth, P.St.J. Russell, M.W. Mason: Supercontinuum generation in submicron fibre waveguides, Opt. Express, vol. 12, pp. 2864-2869, 2004.

[9] A.M. Clohessy, N. Healy, D.F. Murphy, C.D. Hussey: Short low-loss nanowire tapers on singlemode fibres, Electron. Lett., vol. 41, pp. 27-29, 2005.

[10] G. Brambilla, F. Xu, X. Feng: Fabrication of optical fibre nanowires and their optical and mechanical characterization, Electron. Lett., vol. 42, pp.517-518, 2006.

[11] G. Brambilla, F. Koizumi, X. Feng, D.J. Richardson: Compound-glass optical nanowires. Electron. Lett., vol. 41, pp. 400-402, 2005.

[12] L. Tong, J. Lou, Z. Ye, G.T. Svacha, E. Mazur: Self-modulated taper drawing of silica nanowires. Nanotechnology, vol. 16, pp. 1445-1448, 2005.

[13] S. Campopiano, R. Bernini, L. Zeni, P.M. Sarro: Microfluidic sensor based on integrated optical hollow waveguides. Opt. Lett., vol. 29, pp. 1894-1896, 2004.

[14] A.N. Chryssis, S.M. Lee, S.B. Lee, S.S. Saini, M. Dagenais: High sensitivity evanescent field fiber Bragg grating sensor. Photon. Technol. Lett., vol. 17, pp. 1253-1255, 2005. 